\title{
Metodología para Determinar la Distribución de Corrientes de Desbalance en Líneas Aéreas de Transmisión de Energía Eléctrica
}

\author{
Jaramillo, Daniela $^{1^{*} \text { (iD); Fonseca, Antonio }}{ }^{1}$ (D) Pérez, Fabián ${ }^{1}$ (D) \\ ${ }^{I}$ Escuela Politécnica Nacional, Facultad de Ingeniería Eléctrica y Electrónica, Quito, Ecuador
}

\begin{abstract}
Resumen: Las líneas aéreas de transmisión de energía eléctrica presentan un régimen de operación constante y deben soportar esfuerzos mecánicos debido a posibles descargas atmosféricas, en este sentido es importante realizar una adecuada selección de sus conductores de fase y de guarda. Para soportar los esfuerzos mecánicos los conductores de guarda tienen un componente de acero que presenta características eléctricas variables en función de la corriente circulante por el mismo, por otra parte, esta corriente puede determinarse si se conocen los parámetros eléctricos del cable de guarda. En este contexto, el presente documento detalla dos métodos para determinar la distribución de la corriente entre la tierra y el cable de guarda en condiciones de desbalance, con el cual es posible seleccionar los parámetros eléctricos para la modelación adecuada de una línea de transmisión. Los resultados de estos métodos, aplicados en cuatro casos de estudio, se verifican en su respectiva simulación en el software Alternative Transients Program (ATP).
\end{abstract}

Palabras clave. Conductor de acero, Corrientes de desbalance, Líneas de transmisión.

\section{Methodology to Determine the Distribution of Unbalanced Currents of Overhead Electric Power Transmission Lines}

\begin{abstract}
Electrical overhead power lines present a constant function of operation and must withstand against environmental factors such as electrostatic discharges. Therefore, it is important to select adequate phase and ground conductors in order to support their mechanical characteristics. In addition, ground conductors have a steel component presenting variable electrical parameters that can be utilized to determine the current flowing through the ground wire. Accordingly, this document describes two possible methods to determine the distribution of the current between the earth and the ground wire within unbalanced conditions in which it is possible to select the electrical parameters for the correct configuration of a transmission line. The results of these methods, applied in four case studies, can be verified with their respective simulations using the Alternative Transients Program (ATP) software.
\end{abstract}

Keywords. Steel conductor, Unbalanced currents, Transmission lines.

\section{INTRODUCCIÓN}

Las líneas de transmisión son un elemento importante para el transporte de energía, su diseño debe ser realizado cuidadosamente ya que, al operar constantemente, debe estar preparado para cualquier tipo de contingencia o desbalance. El hilo de guarda es un componente importante de la línea, su propósito principal es la protección de los conductores de fase contra la incidencia directa de descargas atmosféricas, que pueden causar solicitaciones eléctricas y mecánicas en la línea, además proporcionan un camino de retorno para las corrientes de cortocircuito.

Bajo estas consideraciones, el conductor de guarda se debe especificar de manera apropiada tanto para conducir las corrientes de cortocircuito pronosticadas del sistema como para el esfuerzo mecánico del conductor ante descargas, es por esa razón que los conductores de guarda generalmente son de acero.
Generalmente cuando ocurre este tipo de fallas en la línea, la corriente de retorno va a circular por tierra y los cables de guarda. La proporción de corriente que va a fluir por cada uno no se la conoce, y es por este motivo que el estudio técnico propuesto presenta dos métodos con los cuales, es posible el cálculo de la cantidad de corriente que posiblemente va a ser transportada por el hilo de guarda, para su selección.

Para realizar este tipo de estudios eléctricos en una línea de transmisión, es necesario usar componentes de secuencia; es por ese motivo que es importante calcular previamente los parámetros eléctricos, y en el caso del hilo de guarda va a depender de las propiedades variables del conductor de acero. Por lo tanto, el estudio técnico va a aplicar dos metodologías para diferentes escenarios con su respectiva simulación en el Software ATP, en donde se puede observar el comportamiento de las corrientes en estado transitorio.

Los métodos son desarrollados a partir del criterio de Kersting (William H. Kersting, 2002) y Blackburn (J. Lewis Blackburn,

danielajaramilloa11@gmail.com

Recibido: 18/07/2019

Aceptado: 10/12/2019

Publicado: 31/01/2020

$10.33333 /$ rp.vol44n2.02 
1993). Los autores han planteado la base para el cálculo de esta corriente, el primero con un análisis a las matrices de las líneas de transmisión y el segundo con un procedimiento diferente al usar el equivalente de las tres corrientes de los conductores de fase con el cable de tierra para formar un circuito monofásico y de esta manera calcular la corriente del hilo de guarda. Gracias a la aportación de la bibliografía mencionada se lleva a cabo este estudio, de tal manera que reúna, desarrolle y compare información, para así validarla y ser usada para estudios futuros.

Además, se detalla un análisis para cables de acero con el cual se puede seleccionar, con cierto porcentaje de error, la impedancia del conductor por ser esta variable con la incidencia de la corriente.

\section{PLANTEAMIENTO DEL PROBLEMA}

La base del estudio es el desconocimiento de la distribución de corrientes de desbalance, ya que estas toman dos caminos: por tierra y por el conductor de hilo de guarda. Hay que resaltar que como parte del estudio se considera el uso de conductor de acero como hilo de guarda, el cual tiene un comportamiento magnético.

\subsection{Corrientes de desbalance}

En condiciones de operación normal las corrientes de desbalance son bajas y cualquier calibre de conductor del cable de guarda puede soportarlas, así que para una correcta selección de cable de guarda se lo determinará mediante corrientes de desbalance ante fallas.

En condiciones de falla, estas corrientes se distribuyen por dos caminos, por tierra e hilo de guarda, la proporción que va a circular por cada uno, no se la conoce. Las corrientes se las puede observar en condiciones transitorias, en este sentido una falla monofásica facilita un análisis adecuado bajo estas condiciones.

\subsection{Conductor de acero}

Cuando se aumenta la corriente que incide en conductores con material de acero, se produce una variación de su permeabilidad magnética, y con ello sus propiedades eléctricas como la resistencia y reactancia interna también van a cambiar, por lo tanto, se puede decir que son conductores con una respuesta variable magnética. Bajo esta condición, la impedancia correcta para este conductor se lo puede determinar conociendo ya la corriente que va a circular por este elemento. Se han realizado varias pruebas en conductores de acero galvanizado y al presentar los resultados de algunos trabajos sobre las propiedades eléctricas de ciertas muestras, que usan composiciones químicas y propiedades mecánicas que ya han sido determinadas previamente, se puede demostrar que cualquier intento de cálculo de los valores de resistencia y reactancia inductiva interna para los conductores de acero es impracticable(E. C. Walton, B. Eng., 1928).

A continuación, en la figura 1 se representa de una forma conveniente las curvas de resistencia y el radio medio geométrico RMG de diferentes tipos de conductores en función de la corriente, además se toma en cuenta que las características son prácticamente proporcionales a la frecuencia es por esta razón que se muestra curvas con 25 y 60 Hertz, para su comparación.

Hay que aclarar que con el valor de RMG se puede calcular la reactancia interna del cable, para la frecuencia de $60 \mathrm{Hertz}$, de la siguiente manera:

$$
x=0,1736 \log _{10} \frac{\text { radio }}{R M G} \Omega / k m
$$

Para 25 Hertz, la constante 0.1736 cambiará a 0.0723 (C. F. Wagner, 1933).
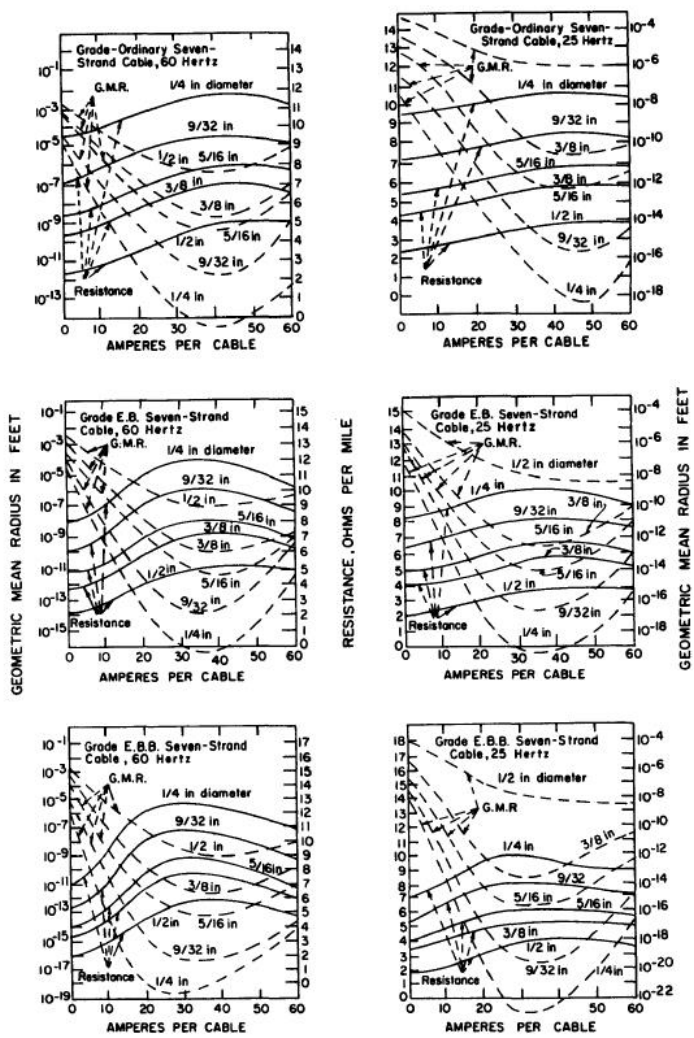

Figura 1. Características eléctricas de los conductores de acero (Paul M. Anderson, 1995).

Con la variación de las características de los conductores ante cambios de la corriente circulante, no se identifican correctamente los parámetros eléctricos de la línea, debido a que se requiere conocer de antemano la corriente que transporta el conductor.

\section{CORRIENTES DE DESBALANCE}

En esta sección se resumen dos metodologías con las cuales son posible el cálculo de la corriente que va a circular por el hilo de guarda. Además, se las comparará con la simulación en el software ATP para su validación.

El modelamiento de la línea ayuda al cálculo de las corrientes de desbalance, por lo tanto, es importante conocer a 
impedancia serie de la línea de transmisión y el efecto de regreso por tierra, de esta manera se usa las ecuaciones de Carson.

El modelo del efecto de retorno por tierra es desarrollado por John Carson en 1926, una publicación que demuestra una técnica para determinar las impedancias propias y mutuas para un número arbitrario de conductores aéreos, considerando el efecto de retorno por tierra. Las ecuaciones pueden ser también aplicadas para cables subterráneos.

Se asume que la frecuencia de 60 Hertz y la resistividad del suelo es de $100 \mathrm{ohm}$-metro, por esto se tienen las siguientes ecuaciones modificadas de Carson(Carson, John R., 1926):

$$
\begin{gathered}
Z_{i i}=r_{i}+0,05923+j 0,0754\left(\ln \frac{1}{R M G_{i}}+7,93402\right) \frac{\Omega}{k m} \\
Z_{i j}=0,05923+j 0,0754\left(\ln \frac{1}{D_{i j}}+7,93402\right) \frac{\Omega}{k m}
\end{gathered}
$$

Además, usando la siguiente relación:

Donde:

$$
7,93402=\ln D_{e}
$$

$D_{e}$ es la profundidad equivalente del regreso por tierra.

Para este valor hay que tomar en cuenta que pueden existir variaciones por el tipo de condiciones de la tierra de retorno. Al estar en función de la resistividad de la tierra $\rho$ y la frecuencia $f$, por esta razón es definida la siguiente relación.

$$
D_{e}=658,35 \sqrt{\frac{\rho}{f}} m
$$

A una frecuencia de $60 \mathrm{~Hz}$ y una resistividad de suelo promedio de $100 \mathrm{ohm}-\mathrm{m}$, se obtiene un valor aproximado de:

$$
D_{e}=850,4 m
$$

Por lo tanto, las ecuaciones de Carson se simplifican de tal manera que se tiene:

$$
\begin{aligned}
& Z_{i i}=r_{i}+0,0592+j 0,0754\left(\ln \frac{D_{e}}{R M G_{i}}\right) \Omega / k m \\
& Z_{i j}=0,0592+j 0,0754\left(\ln \frac{D_{e}}{D_{i j}}\right) \Omega / k m
\end{aligned}
$$

Siendo la Ecuación (4) la impedancia propia de un conductor $i$ y la Ecuación (5) la impedancia mutua entre el conductor $i$ y el conductor $j$.

Con el cálculo de estos valores se puede construir la matriz primitiva de una línea de transmisión como se muestra a continuación (William H. Kersting, 2002).

$$
z_{a b c}=\left[\begin{array}{ccc}
Z_{a a} & z_{a b} & Z_{a c} \\
Z_{b a} & Z_{b b} & Z_{b c} \\
Z_{c a} & Z_{c b} & Z_{c c}
\end{array}\right]
$$

Para encontrar la impedancia de secuencia de la línea se muestra en la Ecuación (7), usando la matriz primitiva de la línea trifásica, sin considerar cable de guarda.

$$
Z_{012}=[A]^{-1} \cdot\left[Z_{a b c}\right] \cdot[A]=\left[\begin{array}{lll}
Z_{00} & Z_{01} & Z_{02} \\
Z_{10} & Z_{11} & Z_{12} \\
Z_{20} & Z_{21} & Z_{22}
\end{array}\right]
$$

Los términos de la diagonal de la matriz son las impedancias de secuencia de la línea de tal manera que:

- $Z_{00}=$ impedancia de secuencia cero

- $Z_{11}=$ impedancia de secuencia positiva

- $Z_{22}=$ impedancia de secuencia negativa

Los términos fuera de la diagonal de la Ecuación (6) representa el acoplamiento mutuo entre las secuencias, en un estado idealizado estos serían cero. Para que esto suceda, una línea de transmisión de alto voltaje debe ser transpuesta. Dado que las líneas de distribución rara vez se transponen, el acoplamiento mutuo entre las fases no es igual, por lo tanto, los términos fuera de la diagonal de la matriz de secuencia serán diferente de cero.

La matriz de impedancia serie de una línea trifásica considerando el hilo de guarda se detalla a continuación:

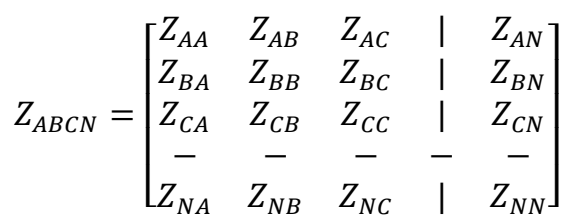

Las líneas de transmisión esencialmente son balanceadas, y es por este motivo que no existen corrientes de secuencia cero. Pero cuando ocurre una falla, el sistema se torna desbalanceado y aparecen estas corrientes llamadas también de desbalance.

Se sabe que en cada fase circulará una unidad de corriente de secuencia cero, y su retorno será la suma de estas tres corrientes.

Generalmente la corriente de retorno se distribuye entre la tierra y el hilo de guarda, sin embargo, no se conoce la proporción que puede circular en este último y es asumida. Parte de esta sección describe como puede ser calculada la corriente que va a circular por el hilo de guarda mediante dos diferentes maneras.

\subsection{Metodología 1: Cálculo de la corriente del cable de guarda con la matriz de impedancias serie (William $\mathrm{H}$. Kersting, 2002)}

Se aplica el método de reducción estándar de Kron, arreglando las matrices de tal manera que se separan las impedancias 
propias de los conductores de fase, del hilo de guarda y las impedancias mutuas entre ellos.

$$
\left[\begin{array}{c}
{\left[V_{a b c}\right]} \\
{\left[V_{n}\right]}
\end{array}\right]=\left[\begin{array}{c}
{\left[V_{a \prime b^{\prime} c^{\prime}}\right]} \\
{\left[V_{n \prime}\right]}
\end{array}\right]+\left[\begin{array}{cc}
{\left[Z_{i j}\right]} & {\left[Z_{i n}\right]} \\
{\left[Z_{n j}\right]} & {\left[Z_{n n}\right]}
\end{array}\right] \cdot\left[\begin{array}{c}
{\left[I_{a b c}\right]} \\
{\left[I_{n}\right]}
\end{array}\right]
$$

Siendo:

$$
\begin{aligned}
& \text { - } \quad i, j \quad \text { número de conductores de fases } \\
& \text { - } n \text { número de conductores de neutro }
\end{aligned}
$$

$\mathrm{Al}$ estar el conductor de guarda aterrizado a tierra, los voltajes $V_{n}$ y $V_{n}$, son igual a cero, sustituyendo estos valores en la Ecuación (9) se tiene las siguientes expresiones:

$$
\begin{aligned}
{\left[V_{a b c}\right] } & =\left[V_{a \prime b \prime c l}\right]+\left[Z_{i j}\right] \cdot\left[I_{a b c}\right]+\left[Z_{i n}\right] \cdot\left[I_{n}\right] \\
{[0] } & =[0]+\left[Z_{n j}\right] \cdot\left[I_{a b c}\right]+\left[Z_{n n}\right] \cdot\left[I_{n}\right]
\end{aligned}
$$

En esta última se despeja la corriente $I_{n}$ con la cual se puede conocer la corriente que posiblemente va a circular por el cable de guarda (William H. Kersting, 2002), en el caso que exista una contingencia en el sistema, es importante conocer las corrientes que van a circular por las fases para realizar el cálculo.

$$
\left[I_{n}\right]=-\left[Z_{n n}\right]^{-1} \cdot\left[Z_{n j}\right] \cdot\left[I_{a b c}\right]
$$

Cada una de las fases lleva una unidad de corriente y su retorno la suma de ellas, para conocer estas corrientes se toma en cuenta que la corriente de retorno será una unidad y que se va a dividir en los tres conductores; y al considerar que la unidad es $100 \%$, cada fase conducirá aproximadamente $33,33 \%$, aplicando lo último en la Ecuación (10), la corriente va a ser expresada en número complejos y para su interpretación se presenta el módulo de este, dejando como resultado el porcentaje de corriente que puede circular a través del hilo de guarda.

Aprovechando estas ecuaciones de la caída de voltaje, queda demostrado cómo se realiza la reducción de la matriz de impedancias, puesto que al tratarse de una línea trifásica con un conductor de guarda tiene dimensiones $4 \mathrm{x} 4$, y para calcular las impedancias de secuencia de la línea es preciso reducir la matriz hasta que sea $3 \times 3$, así que usando las expresiones de la caída de voltaje del circuito se encuentra la ecuación que realiza esta reducción.

$$
Z_{A B C}=\left[Z_{i j}\right]-\left[Z_{i n}\right] \cdot\left[Z_{n n}\right]^{-1} \cdot\left[Z_{n j}\right]
$$

Una matriz primitiva de impedancia que representa los cables de guarda o haz de conductores puede siempre ser convertida en una matriz de $3 \times 3$ y que puede ser usada para calcular de una manera más precisa la caída de voltaje en segmentos de la línea, una vez determinada las corrientes que fluyen a través de las fases.

3.2 Metodología 2: Cálculo de la corriente del cable de guarda con impedancia de secuencia cero(J. Lewis Blackburn, 1993)
Otro método de cálculo es realizando un análisis a la corriente de secuencia cero, como se muestra en la Figura 2, en el que se juntan las corrientes de los conductores de fase, simulando así un conductor equivalente del circuito trifásico, con su respectiva corriente de retorno.
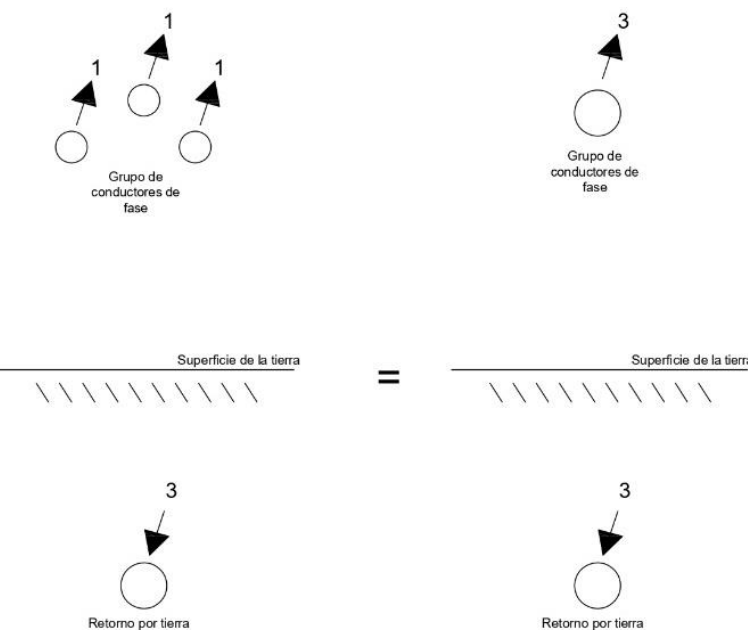

Figura 2. Corrientes de secuencia cero

La red de secuencia cero por definición tendrá una unidad de corriente que consiste en, una unidad de corriente que circula en cada fase del circuito trifásico y tres unidades de corriente de retorno fluyendo por tierra, como se aprecia en la Figura 2. $\mathrm{Al}$ reemplazar las tres fases en un conductor equivalente, las tres unidades de corriente circulan por este.

Usando la ley de Ohm, para mantener la misma caída de voltaje, será lo mismo si una unidad de corriente circula en tres unidades de impedancia; y que tres unidades de corriente fluya en una unidad de impedancia, es por eso qué para mantener la unidad de corriente, esta impedancia es multiplicada por tres(J. Lewis Blackburn, 1993).

Por lo tanto, la impedancia propia y mutua de la línea va a ser multiplicada por tres, para conocer las impedancias de secuencia cero del sistema.

$$
\begin{gathered}
Z_{0}=3 \cdot Z_{A A} \\
Z_{0 m}=3 \cdot Z_{A B}
\end{gathered}
$$

Siendo:

- $Z_{A A}$ impedancia propia de fase de la línea de transmisión

- $Z_{A B} \quad$ impedancia mutua entre los conductores de fase y el retorno por tierra

Con este análisis se tiene la impedancia propia y mutua de secuencia cero de la línea en la Ecuación (12) y Ecuación (13) respectivamente.

$$
\begin{aligned}
& Z_{0 a}=r_{a}+0,1776+j 0,2262\left(\ln \frac{D_{e}}{R M G}\right) \Omega / k m \\
& Z_{0 m}=0,1776+j 0,2262\left(\ln \frac{D_{e}}{D M G}\right) \Omega / k m
\end{aligned}
$$

Incluyendo el cable de guarda, la distribución de corrientes de secuencia cero se distribuyen como se muestra en la Figura 3. 


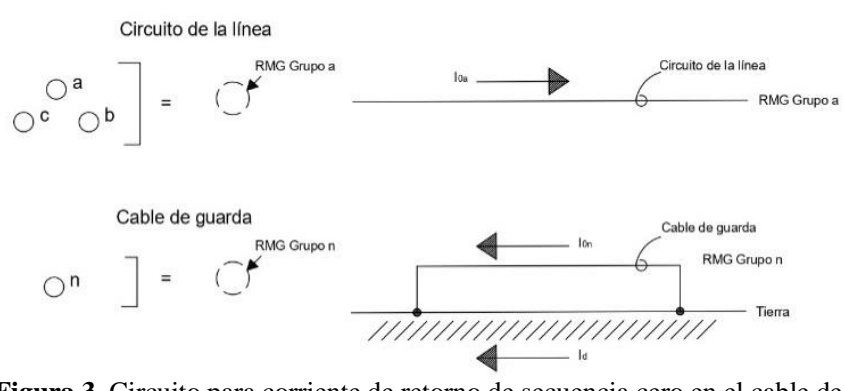

Figura 3. Circuito para corriente de retorno de secuencia cero en el cable de guarda y tierra

Además, se considera ya la impedancia propia de secuencia cero de un cable de guarda que se detalla en la Ecuación (14).

$$
Z_{0 n}=3 r_{n}+0,1776+j 0,2262 \ln \frac{D_{e}}{R M G_{n(\text { gr.de } 1)}}
$$

El $R M G_{g r . a}$ es el radio medio geométrico del circuito de la línea de la Figura 3 que puede ser representado por cualquier tipo de configuraciones de líneas trifásicas, así sea de uno o doble circuito.

Los hilos de guarda están conectados a tierra en cada polo o torre. En la práctica estas conexiones a tierra tendrán la misma impedancia (mayormente resistiva), conocida como resistencia de pie de torre. En los cálculos de impedancia de secuencia cero de la línea, la resistencia de pie de torre es asumida a cero, lo cual es práctico para el cálculo de fallas.

La caída de voltaje a través del circuito de la línea trifásica de la Figura 3 es:

$$
\left[\begin{array}{l}
V_{0 a} \\
V_{0 n}
\end{array}\right]=\left[\begin{array}{ll}
Z_{0 a} & Z_{0 m} \\
Z_{0 m} & Z_{0 n}
\end{array}\right] \cdot\left[\begin{array}{l}
I_{0 a} \\
I_{0 n}
\end{array}\right]
$$

Con estas ecuaciones se tiene el voltaje de secuencia cero de la línea:

$$
V_{0 a}=Z^{\prime}{ }_{0 a} I_{0 a}+Z_{0 m} I_{d}
$$

Donde:

- $Z_{0 a}^{\prime}=Z_{0 a}-Z_{0 m} \quad$ impedancia de dispersión del grupo de conductores de fase

- $I_{d}=I_{0 a}-I_{0 n}$ corriente de retorno que va por tierra

Además, la caída de voltaje en el cable de cero, que será igual a cero.

$$
V_{0 n}=Z_{0 n}^{\prime} I_{0 n}-Z_{0 m} I_{d}=0
$$

Donde:

- $Z_{0 n}^{\prime}=Z_{0 n}-Z_{0 m}$ impedancia de dispersión de los cables de guarda

Con este análisis se obtienen impedancias llamadas de dispersión, en donde anula el efecto mutuo entre los conductores y tierra.

$$
\begin{aligned}
& Z_{0 a}^{\prime}=r+j 0,2262 \ln \frac{\left.D M G_{(g r . d e} 3 \text { a gr.de } 1\right)}{R M G_{a(\text { gr.de } 3)}} \\
& Z^{\prime}{ }_{0 n}=3 r_{n}+j \cdot 0.2262 \cdot \ln \frac{D M G_{(\text {gr.de } 3 \text { a gr.de } 1)}}{R M G_{n(\text { gr.de } 1)}}
\end{aligned}
$$

En la Figura 4 se plasma un circuito equivalente considerando los voltajes de secuencia cero de la línea de la Ecuación (16) y el voltaje del hilo de guarda de la Ecuación (17), para así representar como la corriente de retorno se distribuye entre el cable de guarda y tierra.
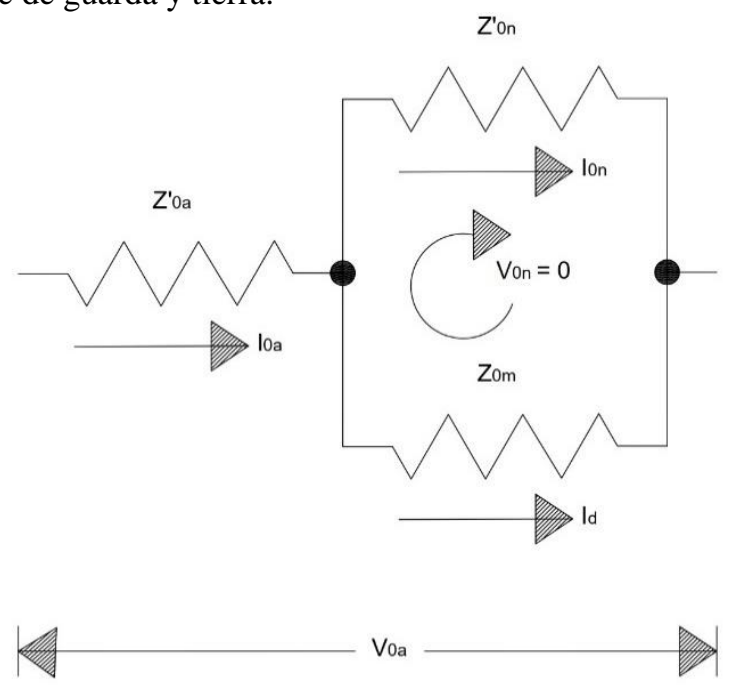

Figura 4. Circuito equivalente para corriente de retorno de secuencia cero a través del cable de guarda y tierra

El cálculo de la impedancia de secuencia cero del circuito se representa en la Ecuación (20).

$$
Z_{0}=Z_{0 a}^{\prime}+\frac{Z \prime_{0 n} Z_{0 m}}{Z \prime_{0 n}+Z_{0 m}}
$$

La proporción de corriente de hilo de guarda se calcula con un divisor de corriente en el circuito de la Figura 4, usando la Ecuación (21).

$$
I_{0 n}=100 \% \cdot \frac{Z_{0 m}}{Z_{\text {on }}+Z_{0 m}}
$$

Para un circuito con dos conductores de guarda, las ecuaciones de impedancias de secuencia cero de este grupo va a cambiar el valor de su resistencia porque la suma en paralelo de los dos conductores va a ser $\mathrm{r} / 2$.

\section{APLICACIÓN DE LAS METODOLOGÍAS}

Las dos metodologías antes descritas serán usadas para calcular cuatro casos de estudios de las referencias (J. Lewis Blackburn, 1993; Paul M. Anderson, 1995).

- Caso 1: una línea trifásica con un cable de guarda, considerando este último como un conductor normal.

- Caso 2: una línea trifásica con dos cables de guarda, considerando estos últimos como conductores normales.

- Caso 3: una línea trifásica con un cable de guarda, considerando este último como un conductor de acero. 
- Caso 4: una línea trifásica con un cable de guarda, considerando estos últimos como conductores de acero.

Se realizarán los cálculos respectivos de forma manual a estos ejercicios, de tal manera que se obtengan los resultados de corriente en el cable de guarda y del valor de la impedancia de secuencia cero.

Los resultados van a ser verificados con el software de simulación ATP (Alternative Transients Program)(László Prikler \& Hans Kristian Hoidalen, 2009). Junto a esto se presenta la configuración del circuito para cada caso.

4.1 Caso 1: línea trifásica con un cable de guarda, considerando este último como un conductor normal

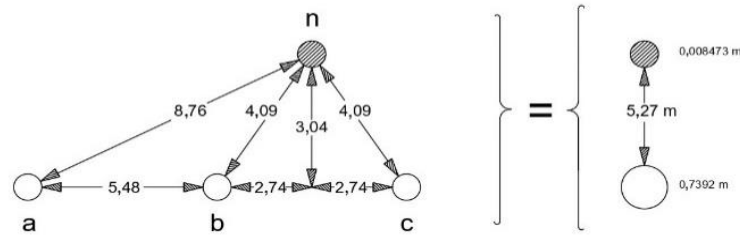

Figura 5. Configuración línea trifásica con un cable de guarda

Tabla 1. Cálculo de error entre las metodologías y la simulación para el primer caso

\begin{tabular}{|c|c|c|c|}
\hline \multicolumn{2}{|c|}{ CASO 1} & $\begin{array}{c}\text { Corriente hilo } \\
\text { de guarda }\end{array}$ & $\begin{array}{l}\text { Impedancia de } \\
\text { secuencia cero }\end{array}$ \\
\hline \multicolumn{2}{|c|}{ Simulación ATP } & $39,74 \%$ & $0,308+\mathrm{j} 1,0938$ \\
\hline \multirow{2}{*}{ Metodología 1} & Valor & $43,27 \%$ & $0,3091+\mathrm{j} 1,0917$ \\
\hline & Error & $8,88 \%$ & $0,15 \%$ \\
\hline \multirow{2}{*}{ Metodología 2} & Valor & $43,10 \%$ & $0,3092+\mathrm{j} 1,0917$ \\
\hline & Error & $8,45 \%$ & $0,15 \%$ \\
\hline
\end{tabular}

El cálculo de la corriente en el hilo de guarda presenta un mínimo error, es por este motivo que se garantiza el uso de estos métodos para un ejemplo tan simple como una línea trifásica con un hilo de guarda, usando el mismo conductor para las tres fases y el hilo de guarda. Además, se puede notar que las impedancias de secuencia cero calculadas también son parecidas entre ellas, y presentan una mínima variación que puede darse por motivos ajenos al procedimiento.

4.2 Caso 2: línea trifásica con dos cables de guarda, considerando estos últimos como conductores normales
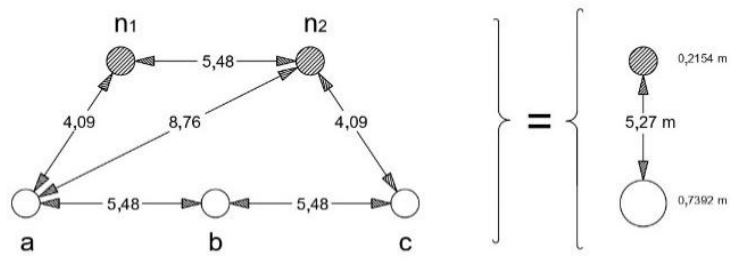

Figura 6. Configuración línea trifásica con dos cables de guarda

Tabla 2. Cálculo de error entre las metodologías y la simulación para el segundo caso

\begin{tabular}{|c|c|c|c|}
\hline \multicolumn{2}{|c|}{ CASO 2} & $\begin{array}{c}\text { Corriente } \\
\text { hilo de } \\
\text { guarda }\end{array}$ & $\begin{array}{l}\text { Impedancia de } \\
\text { secuencia cero }\end{array}$ \\
\hline \multicolumn{2}{|c|}{ Simulación ATP } & $60,07 \%$ & $0,2773+\mathrm{j} 0,8929$ \\
\hline \multirow{2}{*}{ Metodología 1} & Valor & $60,62 \%$ & $0,2776+\mathrm{j} 0,8924$ \\
\hline & Error & $0,92 \%$ & $0,04 \%$ \\
\hline \multirow{2}{*}{ Metodología 2} & Valor & $60,40 \%$ & $0,2778+\mathrm{j} 0,8918$ \\
\hline & Error & $0,55 \%$ & $0,09 \%$ \\
\hline
\end{tabular}

De igual manera para este caso, la corriente no presenta mayor variación, y su error en comparación con la simulación no es mayor al $1 \%$ en ninguno de los dos métodos. La impedancia de secuencia cero de la línea de igual manera tiene un error menor al $1 \%$. La corriente de falla en este caso se va a distribuir por tierra y por los dos cables de guarda que tiene la línea.

\subsection{Caso 3: línea trifásica con un cable de guarda,} considerando este último como conductor de acero

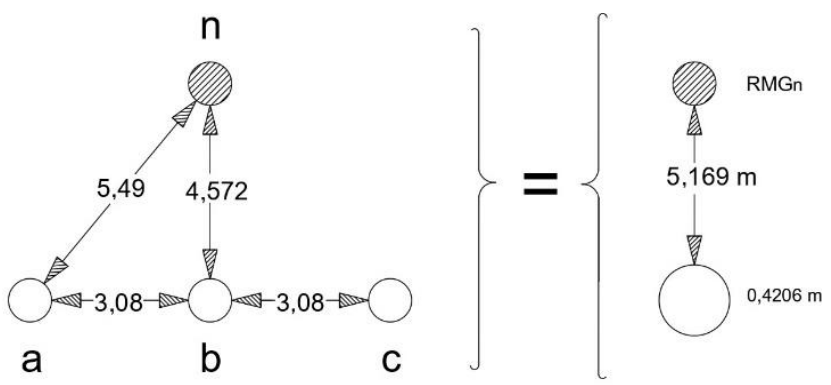

Figura 7. Configuración línea trifásica con un conductor de acero como cable de guarda

Tabla 3. Cálculo de error entre las metodologías y la simulación para el tercer caso, usando impedancia para 1 amperio

\begin{tabular}{cccc}
\hline \multicolumn{2}{c}{ CASO 3 (1 A) } & $\begin{array}{c}\text { Corriente } \\
\text { hilo de } \\
\text { guarda }\end{array}$ & $\begin{array}{c}\text { Impedancia de } \\
\text { secuencia cero }\end{array}$ \\
\hline \multicolumn{2}{c}{ Simulación ATP } & $15,70 \%$ & $0,4789+\mathrm{j} 1,6009$ \\
\multirow{2}{*}{ Metodología 1} & Valor & $15,58 \%$ & $0,4811+\mathrm{j} 1,5948$ \\
& Error & $0,76 \%$ & $0,31 \%$ \\
Metodología 2 & Valor & $14,50 \%$ & $0,4811+\mathrm{j} 1,5948$ \\
& Error & $7,64 \%$ & $0,31 \%$ \\
\hline
\end{tabular}

Tabla 4. Cálculo de error entre las metodologías y la simulación para el tercer caso, usando impedancia para 30 amperios

\begin{tabular}{cccc}
\hline \multirow{2}{*}{ CASO 3 (30 A) } & $\begin{array}{c}\text { Corriente } \\
\text { hilo de } \\
\text { guarda }\end{array}$ & $\begin{array}{c}\text { Impedancia de } \\
\text { secuencia cero }\end{array}$ \\
\hline \multicolumn{2}{c}{ Simulación ATP } & $7,15 \%$ & $0,4042+\mathrm{j} 1,6693$ \\
\multirow{2}{*}{ Metodología 1} & Valor & $7,04 \%$ & $0,4085+\mathrm{j} 1,6633$ \\
& Error & $0,84 \%$ & $0,28 \%$ \\
Metodología 2 & Valor & $6,83 \%$ & $0,4085+\mathrm{j} 1,6633$ \\
& Error & $4,48 \%$ & $0,28 \%$ \\
\hline
\end{tabular}

Tabla 5. Cálculo de error entre las metodologías y la simulación para el tercer caso, usando impedancia para 60 amperios

\begin{tabular}{cccc}
\hline \multirow{2}{*}{ CASO 3 (60 A) } & $\begin{array}{c}\text { Corriente } \\
\text { hilo de } \\
\text { guarda }\end{array}$ & $\begin{array}{c}\text { Impedancia de } \\
\text { secuencia cero }\end{array}$ \\
\hline \multicolumn{2}{c}{ Simulación ATP } & $8,9 \%$ & $0,4141+\mathrm{j} 1,6502$ \\
\multirow{2}{*}{ Metodología 1} & Valor & $8,82 \%$ & $0,4180+\mathrm{j} 1,6443$ \\
& Error & $0,9 \%$ & $0,28 \%$ \\
Metodología 2 & Valor & $8,5 \%$ & $0,4180+\mathrm{j} 1,6443$ \\
& Error & $4,5 \%$ & $0,28 \%$ \\
\hline
\end{tabular}

Se obtiene que en los valores de corriente de hilo de guarda presenta un margen mayor de error con el método que usa impedancias de secuencia cero, valor que no supera el $10 \%$, haciendo efectivo el método. Pero se recomendaría usar el de impedancias de serie, por el hecho de ser más preciso.

4.4 Caso 4: línea trifásica con dos cables de guarda, considerando estos últimos como conductores de acero 


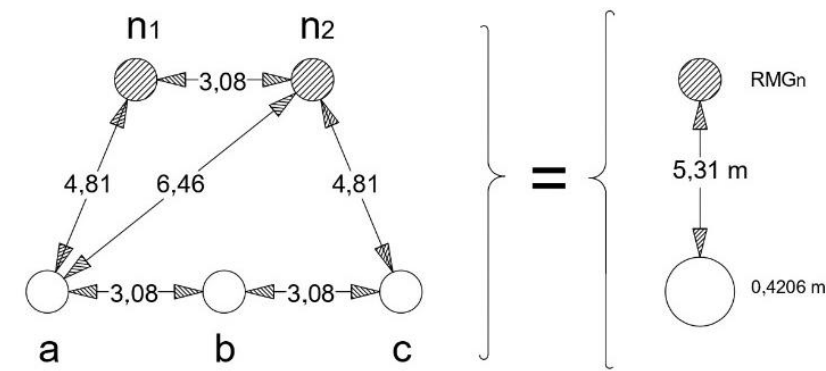

Figura 8. Configuración línea trifásica con dos conductores de acero como cables de guarda

Tabla 6. Cálculo de error entre las metodologías y la simulación para el cuarto caso, usando impedancia para 1 amperio

\begin{tabular}{cccc}
\hline \multirow{2}{*}{ CASO 4 (1 A) } & $\begin{array}{c}\text { Corriente } \\
\text { hilo de } \\
\text { guarda }\end{array}$ & $\begin{array}{c}\text { Impedancia de } \\
\text { secuencia cero }\end{array}$ \\
\hline \multicolumn{2}{c}{ Simulación ATP } & $28,3 \%$ & $0,5521+\mathrm{j} 1,4719$ \\
\multirow{2}{*}{ Metodología 1 } & Valor & $28,02 \%$ & $0,5529+\mathrm{j} 1,4666$ \\
& Error & $0,99 \%$ & $0,29 \%$ \\
\multirow{2}{*}{ Metodología 2 } & Valor & $25,1 \%$ & $0,5529+\mathrm{j} 1,4671$ \\
& Error & $11,31 \%$ & $0,26 \%$ \\
\hline
\end{tabular}

Tabla 7. Cálculo de error entre las metodologías y la simulación para el cuarto caso, usando impedancia para 30 amperios

\begin{tabular}{cccc}
\hline \multirow{2}{*}{ CASO 4 (30 A) } & $\begin{array}{c}\text { Corriente } \\
\text { hilo de } \\
\text { guarda }\end{array}$ & $\begin{array}{c}\text { Impedancia de } \\
\text { secuencia cero }\end{array}$ \\
\hline \multicolumn{2}{c}{ Simulación ATP } & $13,5 \%$ & $0,4500+\mathrm{j} 1,6108$ \\
\multirow{2}{*}{ Metodología 1} & Valor & $13,36 \%$ & $0,4532+\mathrm{j} 1,6050$ \\
& Error & $1,04 \%$ & $0,28 \%$ \\
\multirow{2}{*}{ Metodología 2 } & Valor & $12,68 \%$ & $0,4532+\mathrm{j} 1,6055$ \\
& Error & $6,07 \%$ & $0,25 \%$ \\
\hline
\end{tabular}

Tabla 8. Cálculo de error entre las metodologías y la simulación para el cuarto caso, usando impedancia para 60 amperios

\begin{tabular}{cccc}
\hline \multirow{2}{*}{ CASO 4 (60 A) } & $\begin{array}{c}\text { Corriente } \\
\text { hilo de } \\
\text { guarda }\end{array}$ & $\begin{array}{c}\text { Impedancia de } \\
\text { secuencia cero }\end{array}$ \\
\hline \multicolumn{2}{c}{ Simulación ATP } & $16,4 \%$ & $0,4628+\mathrm{j} 1,5748$ \\
\multirow{2}{*}{ Metodología 1 } & Valor & $16,42 \%$ & $0,4654+\mathrm{j} 1,5692$ \\
& Error & $0,12 \%$ & $0,28 \%$ \\
\multirow{2}{*}{ Metodología 2 } & Valor & $15,52 \%$ & $0,4655+\mathrm{j} 1,5697$ \\
& Error & $5,37 \%$ & $0,25 \%$ \\
\hline
\end{tabular}

En este último caso, los dos métodos llegan a una respuesta con mínimo error comparándola con lo simulado en el software ATP. De igual manera que en el caso 3, se puede observar que donde se tiene un mayor margen de error es en el método dos que usa impedancias de secuencia cero, siendo $11,31 \%$ el error más grande. Hay que resaltar que los cálculos son similares para los conductores de acero, una vez seleccionada la impedancia a usar, en cualquiera de las dos configuraciones, ya sea que se use uno o dos hilos de guarda.

\section{ANÁLISIS DE LOS CONDUCTORES DE ACERO}

En esta sección se muestra el análisis del comportamiento del conductor de acero como hilo de guarda en una línea de transmisión, para esto se hace uso del simulador ATP en donde se ha manipulado de tal manera que se puede observar las formas de onda de corriente de falla y del hilo de guarda, para así compararlas y poder observar que proporción de corriente va a retornar por el cable de guarda.
Hay que destacar que se ha simulado el caso tres por ser un circuito simple que usa solamente un hilo de guarda, y para conocer las características eléctricas de este último conductor usando los datos de la figura 1 , se modificó el circuito de tal manera que la corriente que circule por el hilo de guarda se encuentre dentro del rango de 1 a 60 amperios, y con esto realizar el proceso iterativo que el libro menciona como solución al problema, el cual establecerá una impedancia precisa para el conductor de acero. Este método consiste en tomar una impedancia y calcular su corriente, con esta última magnitud compararla con las curvas y encontrar la impedancia adecuada. Tomando en cuenta lo dicho anteriormente por la referencia para encontrar los valores de resistencia y de reactancia del conductor de acero, se realiza de una manera iterativa, se toma una impedancia de las curvas, como ejemplo se iniciarán con los valores que se obtienen para 1 amperio, y como resultado de la corriente de falla se tiene que es 62,68 amperios, que sobrepasa el rango, pero su valor cercano es de 60 amperios por lo que se toman estos valores de impedancia y se simula nuevamente con estos datos, dando como resultado la corriente de 31,93 amperios. Y otra vez se toman los valores de resistencia y reactancia para esta última corriente que por la forma de curva serán similares a los de 30 amperios, y la corriente resultante en la simulación será de 28,7 amperios.

Por lo tanto, para este caso, se tendrá una resistencia de 4,84 ohmios por kilómetro y un radio medio geométrico de $1,5 \mathrm{E}-12$ metros, que es una reactancia 1,9624 ohmios por kilómetro.

Tabla 9. Datos de conductor de acero tipo EBB-0,9525 centímetros con corrientes simuladas para cada impedancia

\begin{tabular}{ccccc}
\hline Corriente & Resistencia & $\begin{array}{c}\text { Reactancia } \\
\text { propia }\end{array}$ & \multicolumn{2}{c}{$\begin{array}{c}\text { Corriente hilo de } \\
\text { guarda }\end{array}$} \\
\hline $\mathrm{A}$ & $\Omega / \mathrm{km}$ & $\Omega / \mathrm{km}$ & $\mathrm{A}$ & $\%$ \\
1 & 2,175 & 0,520 & 62,68 & $15,72 \%$ \\
10 & 3,107 & 1,041 & 44,20 & $11,02 \%$ \\
20 & 4,350 & 1,736 & 31,61 & $7,88 \%$ \\
30 & 4,847 & 1,962 & 28,70 & $7,16 \%$ \\
40 & 4,661 & 1,962 & 29,57 & $7,37 \%$ \\
50 & 4,226 & 1,918 & 31,76 & $7,92 \%$ \\
60 & 3,729 & 1,705 & 31,93 & $7,96 \%$ \\
\hline
\end{tabular}

Se observa que la proporción de corriente del hilo de guarda no presenta mayor variación entre las impedancias dadas desde los 20 hasta los 60 amperios, así que se podría concluir que para este conductor se puede escoger una impedancia dentro del último rango mencionado, ya que tiene valores de resistencia y reactancia propia con menor porcentaje de error respecto a la impedancia que se ha escogido mediante el método iterativo.

El error para cada impedancia es calculado en la Tabla 10, un analista de falla puede escoger, dentro de rango seleccionado de esta última tabla, la impedancia para 30 amperios y considerar así un error máximo de $13 \%$ para los valores de reactancia (no se toma en cuenta el cálculo de error del radio medio geométrico ya que los datos se grafican en escala logarítmica y son muy pequeños por lo tanto no da un valor de error exacto) y en el caso de la resistencia será $23 \%$ de error. 
Tabla 10. Cálculo de error de impedancia para conductor de acero tipo EBB0,9525 centímetros respecto a los valores de resistencia y reactancia escogidos

\begin{tabular}{ccc}
\hline & RESISTENCIA & REACTANCIA \\
\hline \% Error 1 A & $55.13 \%$ & $73.45 \%$ \\
$\%$ Error 10 A & $35.90 \%$ & $46.91 \%$ \\
$\%$ Error 20 A & $10.26 \%$ & $11.51 \%$ \\
$\%$ Error 30 A & $0.00 \%$ & $0.00 \%$ \\
$\%$ Error 40 A & $3.85 \%$ & $0.00 \%$ \\
$\%$ Error 50 A & $12.82 \%$ & $2.26 \%$ \\
$\%$ Error 60 A & $23.08 \%$ & $13.07 \%$ \\
\hline
\end{tabular}

Para mayor comprensión del método se muestra el diagrama de flujo en la Figura 9.

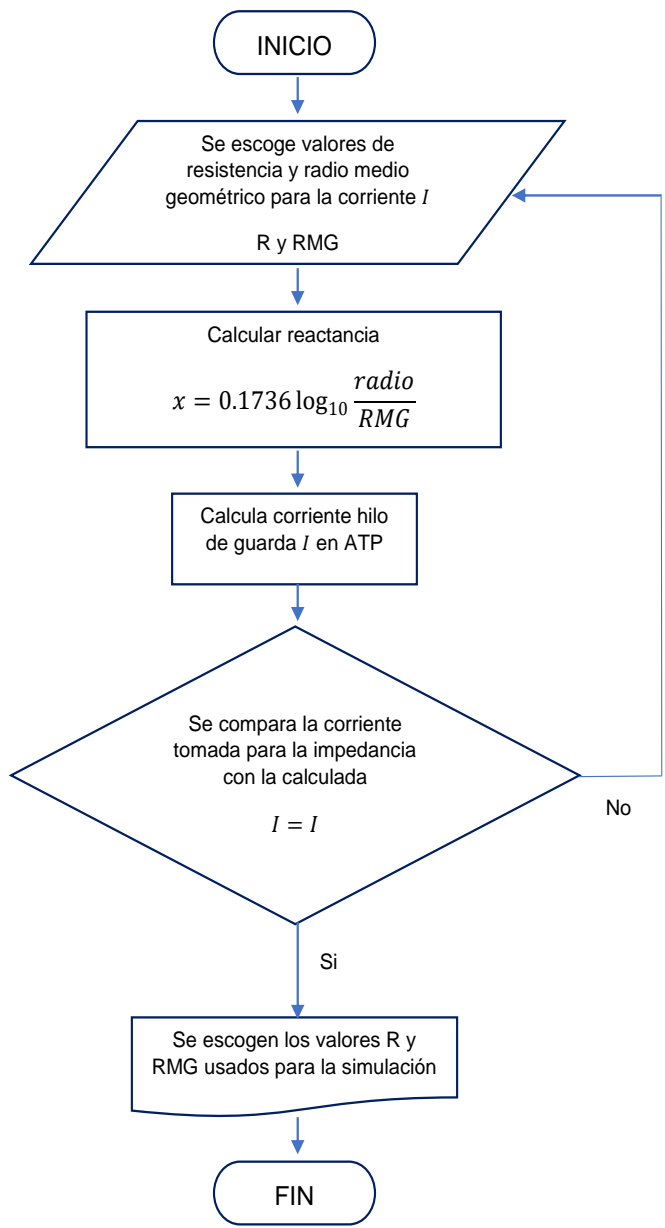

Figura 9. Diagrama de flujo para buscar la impedancia adecuada del conductor de acero

Es importante el uso del simulador ATP ya que, al ser un método iterativo, como se muestra en la Figura 9, es de gran ayuda para el cálculo de corriente para encontrar la impedancia adecuada del conductor de hilo de guarda.

\section{CONCLUSIONES}

Con las metodologías indicadas en el presente documento, es posible determinar las corrientes de desbalance que van a circular por el hilo de guarda, además, estas metodologías permiten analizar el comportamiento del conductor de guarda usando conductores de material de acero con sus características eléctricas variables.

El método de impedancia de serie de línea utiliza las matrices primitivas de impedancia de la línea de transmisión, en donde se incluye el efecto de corriente de retorno a través de la tierra usando ecuaciones de Carson que calcula las impedancias mutuas y propias de la matriz primitiva, considerando que la tierra es un sólido infinito con una superficie uniforme, plana y con resistividad constante.

El método de impedancias de secuencia cero utiliza fórmulas que agrupan a todos los conductores de fase y cables de guarda respectivamente. Las impedancias de estas son propias sin considerar el efecto de retorno a tierra, en cambio la impedancia mutua depende de este efecto, y con la ayuda de estos datos se construye un circuito equivalente donde se observa la distribución de corriente de falla entre la tierra y el cable de guarda.

La resistencia y reactancia propia interna de los conductores con material magnético, como el acero, no es constante ya que presenta variación en la permeabilidad magnética al momento de incrementar la corriente en el cable.

En el caso de conductores de acero, al no conocer su impedancia con exactitud, se establece un posible procedimiento iterativo, donde usando ciertos valores de resistencia y reactancia se calcula la corriente que puede fluir por el cable, y con este valor buscar los valores de impedancia adecuados. Complementariamente, la determinación de la corriente se lo realiza en ATP permitiendo verificar, mediante simulación, los resultados encontrados con el procedimiento iterativo propuesto en este documento

El documento contiene información de varios autores que fue desarrollada y comprobada entre sí, además es de gran importancia para realizar trabajos futuros sobre este tema.

\section{REFERENCIAS}

C. F. Wagner, R. E. (1933). Symmetrical Components (First Edition). New York: McGraw-Hill Book Company, Inc.

Carson, John R. (1926). Wave Propagation in Overhead Wires wtih Ground Return. Bell System Technical Journal, 5, 539-554.

E. C. Walton, B. Eng. (1928). THE ELECTRICAL PROPERTIES OF GALVANIZED STEEL CONDUCTORS FOR OVERHEAD TRANSMISSION LINES. Journal of the Institution of Electrical Engineers, 66(382), 1065-1078.

J. Lewis Blackburn. (1993). Symmetrical Components for Power Systems Engineering. New York: Marcel Dekker, Inc.

László Prikler, \& Hans Kristian Hoidalen. (2009). ATPDRAW User's Manual. Paul M. Anderson. (1995). Analysis of Faulted Power Systems. New York: Power Math Associates, Inc.

William H. Kersting. (2002). Distribution System Modeling and Analysis. Las Cruces, New Mexico: CRC Press. 


\section{BIOGRAFÍAS}

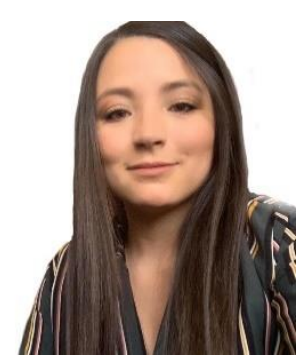

Daniela Jaramillo, nació el 11 de mayo de 1993 en Ibarra, Ecuador. Culmino el bachillerato en ciencias, especialidad Físico Matemático en la Unidad Educativa "Sagrado Corazón de Jesús" Hnas. Bethlemitas. Se graduó recientemente de Ingeniera Eléctrica en la Escuela Politécnica Nacional.

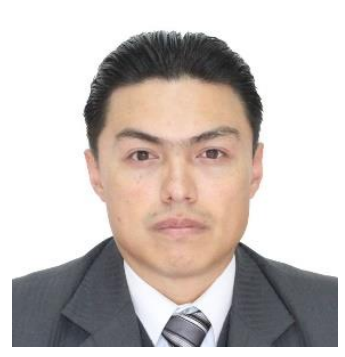

Antonio Fonseca, nació en Quito, Ecuador en 1979. Recibió su título de Ingeniero Eléctrico (marzo 2003) y Magister en Ingeniería Eléctrica (julio 2009) en la Escuela Politécnica Nacional (EPN) QuitoEcuador. Actualmente, forma parte del Departamento Mantenimiento de CELEC EP - Unidad de Negocio TRANSELECTRIC. Adicionalmente se desempeña como profesor en la carrera de Ingeniería Eléctrica y en la Maestría en Ciencias de Ingeniería Eléctrica de la EPN.

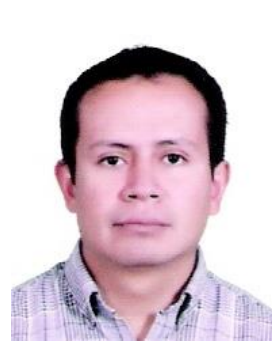

Fabián Pérez Yauli, nació en AmbatoEcuador. Obtuvo el título de Ingeniero Eléctrico en la Escuela Politécnica Nacional, Quito-Ecuador en 2004. En 2012 obtuvo el grado de Doctor en Ingeniería Eléctrica en la Universidad Nacional de San Juan, San JuanArgentina, con una beca otorgada por el Servicio Alemán de Intercambio Académico (DAAD). Actualmente es profesor titular a tiempo completo en la Escuela Politécnica Nacional. Su rama de investigación incluye protecciones de sistemas de potencia y procesamiento de señales. 
